\title{
Implantable Electronic Devices Technology Challenges for Long-Term Human Implantation
}

\author{
A. Vanhoestenberghe
}

\begin{abstract}
Purpose To discuss the requirements for long-term implantation of electronic devices with a focus on packaging and encapsulation.

Approach Due to their intended long-term use in the human body, implants for electrical stimulation present specific challenges to the engineers. The respective roles of packaging and encapsulation must be clearly understood to make the most of new materials and modern machining technologies. This paper offers an introduction to the current situation and highlights challenges for future developments.

Findings The innovative application of modern technologies may be usefull to tackle key issues of encapsulation and sealing of small electrical devices for long term implantation.

Originality Two examples of innovative application of alternative package manufacture and sealing method are described.
\end{abstract}

Keywords Hermetic packaging, encapsulation, functional electrical stimulation, implanted devices.

Paper type Research paper.

\section{Introduction}

Implantable electronic devices present specific challenges to the designers due to their long-term intended use within the human body. The development of the first such devices in the late 1950s resulted from a combination of two fundamental technological advances. On the one hand, the reliability of electronic devices working in hostile environment had greatly improved during the first half of the century with major progresses in packaging. On the other hand, pioneers in neurophysiology and medical engineering had raised the general understanding of the human neuromotor system and how to interact with it. This led to considerable innovations in the use of electronic devices for medical applications, with the early percutaneous heart pacemakers appearing in 1952 and the first implantable version in 1958. Fifty years on the technologies have evolved and seen the successful commercialisation of a variety of electrical stimulation implants. Yet the production processes are complex, the materials used are expensive and the need for CE/FDA approval imposes rules on the design, some of which seem to stem less from reasonable investigation than from an unfortunate lack of understanding of the long-term effects of human implantation. This paper discusses some of the technologies available to small research groups for the production of human implants, with a focus on the challenges for packaging and encapsulation.

\section{A Implant parts}

Most electrical stimulation implants are seen as the combination of their two fundamental components: an electronic circuit to produce the stimuli, and electrodes to deliver the charges to the nervous structures. These two parts are connected to one another with a flexible cable. The circuit may be enclosed in a hard shell, the package, while the assembly (with or without packaging) may also be coated in a soft shell or encapsulation.

\section{B Implants requirements}

Integrity is essential, consistency of the electronic function must be guaranteed over the expected life-time and under all unexpected conditions. No leakage current must flow through the electrodes. Corrosion is the main concern due to the humid atmosphere and emphasis is often placed on the hermeticity of the package and its seal which may be tested and monitored. The encapsulant also 


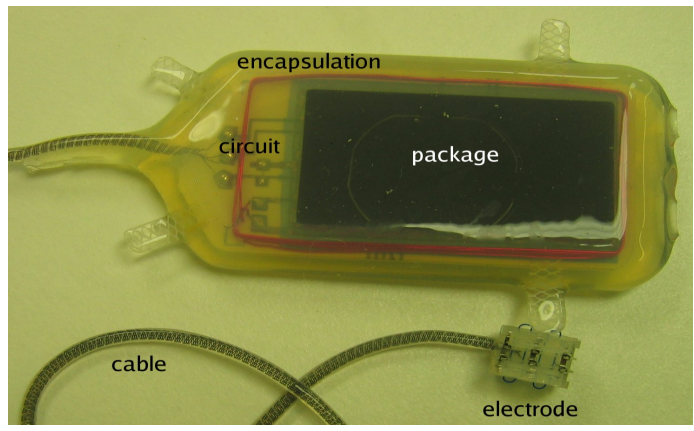

Figure 1: The 5 parts of an implant: the encapsulation, the circuit mainly hidden under the package, the cable and the electrode.

plays a role in preventing corrosion of all metallic areas that cannot practically be encased. While the package and encapsulant protect the circuitry from the adverse environment, biocompatibility ensures that the host is not endangered by the device. All materials in contact with the body, i.e. the metal of the electrodes, the packaging and the encapsulant, must be tested for adverse tissue reactions. Mechanically, parts of the device may be subjected to considerable strain. They must be robust to withstand the flexion and wear, yet soft and smooth to prevent damaging the body.

\section{Materials and methods.}

\section{A Packaging}

Various biocompatible materials have been used over the years, mainly glass, ceramic $\left(\mathrm{Al}_{2} \mathrm{O}_{3}\right)$ and Titanium. The later is acceptable as it is protected from corrosion by a strongly passivating oxide layer and is widely used in passive implants such as dental implants, joint prostheses and bone fixation screws as well as electrical stimulator enclosures. The main issues are machinability of the materials to create the case, the formation of feedthroughs, the need to accommodate for a radio antenna and the sealing process. The enclosure must not alter the communication with the implant controller (carried outside the body), often established through a radio-frequency link, and the presence of a shorted metal loop may considerably decrease the performances. The need to machine intricate shapes and the trend towards miniaturisation further increases the difficulties in finding suitable materials and providing adequate feedthroughs and sealing methods. Prototyping and rapidity of alterations are essential for small research facilities so new packaging are under study that can be produced and sealed on-site to limit the reliance on subcontractors and cut costs. These developments must be undertaken in parallel with the circuit design as sealing and feedthroughs will influence the method chosen to produce the circuit. Advances in the development of rechargeable batteries makes it a reasonable aim to include a power source inside the implant rather than rely on an external battery pack powering the implant by induction $(\mathrm{MHz})$. This adds further constraints on the implant design as some batteries, and other new components, will be destroyed at temperatures well below that used in common joining processes used to seal the package, such as soldering, brazing and anodic bonding.

\section{B Encapsulation}

Often neglected or poorly understood, the encapsulant is essential for long term implantation. It provides a soft and smooth coating for packages presenting sharp edges and dangerous corners that could cause cuts and bleeding within the body. Silicone rubber, the most common encapsulant, has a long history of implantation and its toxicity records are well-known. Further to its padding properties, its main function on the implant is to prevent corrosion of all coated metal parts by avoiding direct contact with body fluid. Yet it is no barrier to water. Indeed, once implanted, the encapsulation layer becomes quickly saturated with water (a couple of days for a $5 \mathrm{~mm}$ thick coat). If water was to condense in a void or gap between the encapsulant and the substrate, the presence of a salt (residue after cleaning stage or corrosion product) could have dramatic consequences. It would dissolve into a highly-concentrated solution and depending on its solubility, osmotic pressure may drive more water inwards, leading to further loss of adhesion and failure [Donaldson, 1989, 1991].

The encapsulant is complementary to the package. It protects the seal, the feedthroughs and the metal connection pads (for electrodes and antenna) from corrosion. It is therefore critical to study the adhesion properties between any encapsulant candidate and the other materials of the implant and to design the implant and its package with care to prevent the formation of voids during the moulding and curing process. Although it is not a barrier to water, silicone rubber may stop ions from propagating from the package into the body fluid. This offers the option to use a wider variety of metals and further study is necessary to determinate the biocompatibility of other combinations of package materials and encapsulant.

\section{Challenges}

The production of all five parts of an implant may benefit from modern technologies. Schuettler and 
Suaning have successfully established a novel electrode fabrication method using a high precision engraving laser [Schuettler et al., 2005]. The Implanted Devices Group has long been a pioneer in hermetic packaging and encapsulation [Donaldson, 1988, 1987]. The actual challenges in these domains are: simplify assembly with low temperature sealing and new machining methods, improve prototyping capabilities and response time through faster processes and reduced reliance on external subcontractors, prevent obsolescence and reduce cost by investing research effort in new materials.

\section{Examples}

In this section two alternative packaging methods developed at the Implanted Devices Group are presented.

\section{A Laser soldering of lid to control inner temperature}

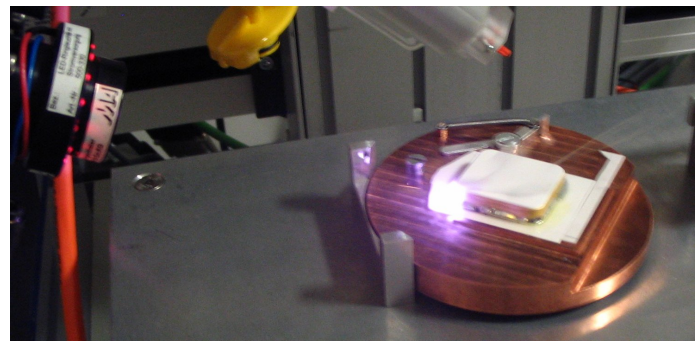

(a) Laser soldering of alumina lid to HTCC package.

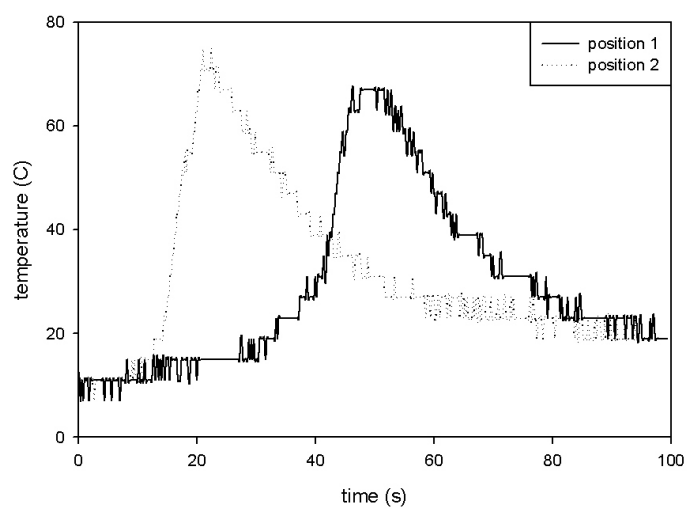

(b) Temperature inside the package during laser soldering

Figure 2: Laser soldering.

One challenge when using the current packaging methods is that they all require relatively high temperatures which could result in permanent damages of the components being encased. During the development of a remote controlled stim- ulator implant we were asked to produce a prototype including a rechargeable battery whose maximum temperature was specified as $80^{\circ} \mathrm{C}$. The solution proposed uses a laser beam to locally heat the solder and propagate the melted front around the seal fast enough to prevent excessive heating at the center of the package. During a sealing run, the temperature was monitored in two places inside the package, corresponding to the centre and one corner of the battery area closer to the lid being soldered. The results are presented in figure 2 (b) with a picture of the laser soldering in progress 2(a). Despite the slow rotating speed required to ensure a smooth continued fillet, the temperature did not reach the critical $80^{\circ} \mathrm{C}$.

\section{B Electroformed very small lids.}

For this project the challenge was to produce a package that could be implanted directly next to the electrode-cuff. To limit the damages and risks of pulling on the nerve after the electrode placement, it was suggested to mount the package on the cuff if it could be made small enough. As a prototype the following solution was implemented, see figure 3. The package is made of a thin $(254 \mu \mathrm{m})$ alumina substrate with multiple layers thick film tracks and dielectric, and a copper lid, electroformed on a mandrel machined out of steel then hardened. This method allowed the production of small lids with very thin walls $(300 \mu \mathrm{m})$ and a particular shape (there is an overhang on two sides of the lid to be soldered on the other side of the substrate). The lids are then soldered using a standard reflow oven. Hermeticity was tested using a helium leak detector to measure the rate of helium escaping the package after bombing it with helium under pressure for $48 \mathrm{~h}$. Although this procedure is not ideal it gives a first indication of the quality of the seal. Further tests will follow as the results of this preliminary evaluation were successful. Copper is not a standard material for human implant but this solution offers the possibility to quickly produce very small lids with dedicated shapes than can easily be altered. The bio-toxicity of the metal is not an issue for the short-term tests intended in this project as corrosion will be prevented by the encapsulant.

\section{Discussion}

Titanium cases or glass enclosures anodically bonded to silicon wafers are suitable packaging methods for implantable electronic devices. Yet the production processes are complex and the difficulty to create feedthroughs for the electrodes renders these option rather unreasonable for small research groups. Alternative methods such as 


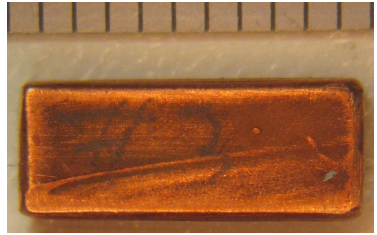

(a) Electroformed copper lid

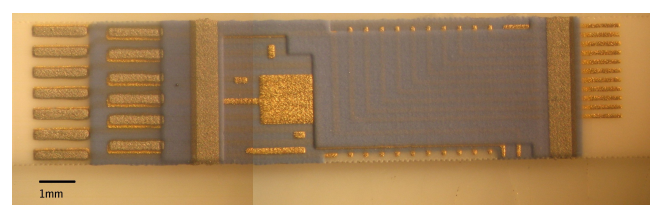

(b) Thick-film printed substrate.

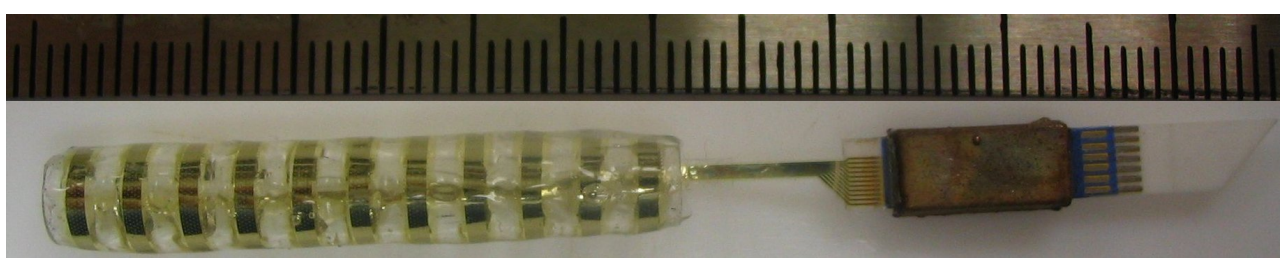

(c) Copper lid mounted on thick film substrate with thin-film Pt electrode.

Figure 3: Fine electroformed copper lids.

$\mathrm{Al}_{2} \mathrm{O}_{3}$ lids glued and soldered onto alumina substrates have been in use for more than 20 years now. The circuit and feedthroughs density may be increased when moving from screen-printed gold and platinum tracks on $\mathrm{Al}_{2} \mathrm{O}_{3}$ substrates to HTCC but this improvement comes as a compromise with increased the cost and lowered versatility. Work is ongoing in developing packages that may be produced on site, requiring a limited set of machines and allowing rapid prototyping an easy design alterations. A proper understanding and recognition of the role of the encapsulant is necessary to keep realistic implant design proposals from being abandoned due to misguided interpretation of official approval rules.

\section{Conclusion}

How can we make the most of today's technologies? Current research into alternative materials for encapsulation, packaging, electrodes and circuit tracks together with original applications of micro-machining methods will simplify the production process with high yield and reliability, leading to smaller implants, increased functionalities and lower costs.

\section{Acknowledgements}

The author wishes to thank Prof Donaldson, and the European Union (Healthy Aims) and the EPSRC who funded projects during which the developments presented in this paper were undertaken.

\section{References}

Donaldson N. de N. Low-technology sealing method for implantable hermetic packages.
Med. Biol. Eng. Comput., 26:111-116, 1988.

Donaldson P.E.K. Twenty years of neurological prosthesis-making. J. Biomed. Eng., 9 (October):291-298, 1987.

Donaldson P.E.K. Encapsulating microelectronic implants in one-part silicone rubbers. Med. Biol. Eng. Comput., 27:93-94, 1989.

Donaldson P.E.K. Aspects of silicone rubber as an encapsulant for neurological prostheses - part 1: Osmosis. Med. Biol. Eng. Comput., 29:34-39, 1991.

Schuettler M., Stiess S., King B.V., and Suaning G.J. Fabrication of implantable microelectrode arrays by laser cutting of silicone rubber and platinum foil. J. Neural Eng., 2:S121-S128, 2005. 